\title{
MRI of Cerebellar Infarction
}

\author{
Laurens J.L. De Cocker ${ }^{\mathrm{a}}$ b Karl-Olof Lövblad ${ }^{c}$ Jeroen Hendrikse ${ }^{\mathrm{a}}$ \\ a Department of Radiology, University Medical Center Utrecht, Utrecht, The Netherlands; ${ }^{\mathrm{b}}$ Department of Radiology, \\ Kliniek Sint-Jan, Brussels, Belgium; ' Department of Radiology, Geneva University Hospitals, Geneva, Switzerland
}

\section{Key Words}

Advanced MRI - Cerebellar infarctions .

Diffusion MRI - diffusion-weighted imaging • Magnetic resonance perfusion - Neuroimaging in stroke $\cdot$ Silent infarct · Stroke imaging · Structural MRI neuroimaging · Vertebrobasilar disease - Venous sinus thrombosis

\begin{abstract}
Background: MRI is the imaging modality of choice for diagnosing brain infarction. Because of few or atypical clinical symptoms and a relatively low sensitivity of CT scans, many cerebellar infarctions may be detected only with MRI. With adequate recognition of cerebellar infarction on MRI and prompt initiation or optimisation of preventive therapeutic measures, more dramatic strokes may be avoided in selected cases. Summary: We first briefly review the clinical presentation of cerebellar infarctions, followed by a short refresher on cerebellar anatomy and pathophysiological mechanisms of cerebellar infarcts. Then, we review the arterial cerebellar perfusion territories recently made visible with territorial arterial spin labeling (ASL), followed by a discussion and illustration of the MRI appearance of cerebellar infarcts in different stages. Similar to large cerebellar infarcts, recent studies investigating volumetric MRI datasets have now shown that small cerebellar infarcts occur in typical spatial patterns, knowledge of which may help in the diagnosis of even the smallest of cerebellar infarcts on MRI. Key Messages: MRI is the modality of choice for diagnosing cerebellar infarction. The posterior inferior cerebellar artery (PICA)-territories can be visualised with super-selective territorial ASL MRI. The
\end{abstract}

\section{KARGER}

(c) 2017 S. Karger AG, Basel

E-Mail karger@karger.com

www.karger.com/ene
PICA supplies at least the medial part of the posterior cerebellar surface. Anterior inferior cerebellar artery-infarcts can be mistaken for lateral PICA-infarcts. Small infarcts typically affect the cortex and often present as incidental cavities. Subacute cerebellar infarcts may be missed on imaging due to a phenomenon called "fogging."

(c) 2017 S. Karger AG, Basel

\section{Introduction}

Cerebral infarcts on MRI are no longer a diagnostic challenge, mainly thanks to the strength of diffusionweighted imaging (DWI) and fluid attenuated inversion recovery (FLAIR) imaging [1-3]. In the cerebellum, however, FLAIR images are less robust due to magnetic field heterogeneities in the posterior fossa $[4,5]$, while nonfocal clinical symptoms and delays in MRI often lead to imaging outside the time frame of diffusion restriction [6]. This way, recent studies have indicated that most cerebellar infarcts initially remain unnoticed, and are only detected later on as an incidental infarct cavity on CT or MRI $[7,8]$. Although patients diagnosed with cerebellar infarction on imaging often lack a clinical history of vertebrobasilar transient ischaemic attack (TIA) or stroke, they carry an increased risk of recurrent ischaemic events $[7,8]$. Prompt diagnosis of cerebellar infarctions with the aid of MRI is thus warranted to prevent future stroke.

In this article, we briefly review the clinical presentation of cerebellar infarctions, followed by a short review of cerebellar anatomy and an update on cerebellar infarct 

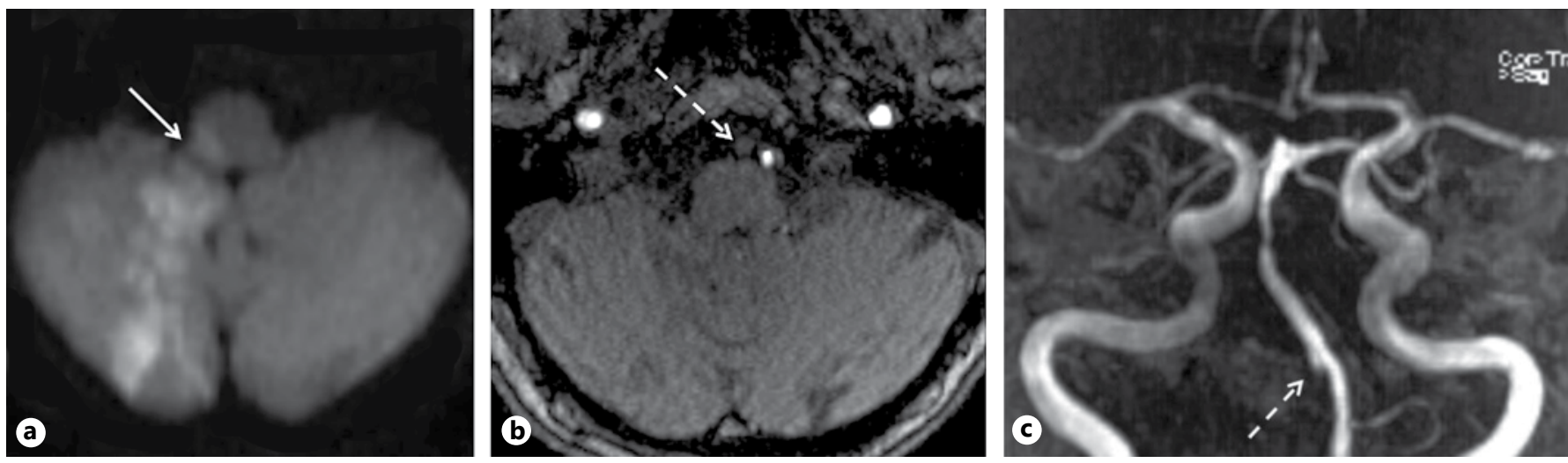

Fig. 1. Acute PICA infarct (arrow) involving the medial part of the posterior lobe of the cerebellum as well as the adjacent posterolateral medulla, as seen on DWI (a) in a patient with lateral medullary

pathophysiology. We then review and illustrate the cerebellar arterial perfusion territories and the appearance of cerebellar infarcts as presented by MRI.

\section{Clinical Presentation}

Cerebellar infarcts typically present with non-specific symptoms such as dizziness, nausea, vomiting, unsteady gait and headache [6]. It may mimic benign conditions such as viral gastroenteritis or labyrinthitis, which are often seen in patients admitted to the emergency department [9]. Neurological signs associated with cerebellar infarcts include dysmetria, dysarthria, ataxia and nystagmus [10]. A lateral medullary syndrome or Wallenberg syndrome may be seen with posterior inferior cerebellar artery (PICA) infarctions (Fig. 1).

In general, small cerebellar infarcts present with the same though less severe and less profound clinical manifestations as compared to larger infarcts [11]. As a result of subclinical cerebellar infarctions, small incidental infarct cavities are a very frequent finding in the cerebellum, especially in ageing subjects and patients with cardiovascular disease, usually without a known history of cerebellar TIA or stroke $[7,8]$.

\section{Anatomy}

\section{Structural Anatomy}

The cerebellum is located in the posterior fossa of the skull and consists of a midline vermis and 2 lateral hemispheres. It has 3 surfaces; an anterior (or petrosal), supe- or Wallenberg syndrome. TOF-MRA images $(\mathbf{b}, \mathbf{c})$ show occlusion of the right vertebral artery (dashed arrows) and right PICA, while the left PICA can still be observed (c). rior (or tentorial) and posterior (or occipital) surface. The cerebellum is composed of 3 lobes - the anterior lobe, the posterior lobe and the flocculonodular lobe [12]. The anterior lobe is separated from the posterior lobe by the primary fissure, which is easily identified in the axial and midsagittal plane as the deepest and thickest fissure in the superior part of the vermis. The flocculonodular lobe is composed of the nodule of the vermis in the midline and of the flocculus in the cerebellar hemispheres.

\section{Arterial Anatomy and Perfusion Territories}

The cerebellum mainly receives arterial blood supply from 3 paired cerebellar arteries, although variant arterial anatomy is common, including duplication, double origin, and the absence or dominance of a cerebellar artery. The PICA usually arises from the distal vertebral artery and supplies the posterior surface of the cerebellum, at the least in its medial portion (Fig. 1-3) [13, 14]. The anterior inferior cerebellar artery (AICA) arises from the first or second or third part of the basilar artery, and it often demonstrates a loop in the inner auditory canal (Fig. 4). The superior cerebellar artery arises near the termination of the basilar artery and typically supplies the superior surface and anterior lobe of the cerebellum (Fig. 5).

The 3 cerebellar arteries also take part in the vascularisation of the brainstem (Fig. 1) [14]. The cerebellar arteries give rise to medial and lateral branches, of which the medial branch supplies the lateral and posterior brainstem arterial territories [14]. The PICA takes part in the lateral and posterior arterial groups of the medulla (Fig. 1) [14]. The AICA supplies the middle cerebellar peduncle and often the lower part of the pontine tegmentum, while 
Fig. 2. Variability of the PICA perfusion territory demonstrated with super-selective territorial ASL MRI in 10 healthy subjects. The PICA invariably supplies the medial parts of the posterior inferior surface, with variable extension towards the lateral side of the posterior surface as well as variable extension towards the superior and anterior cerebellar surfaces. Reproduced from Hartkamp et al. [13].

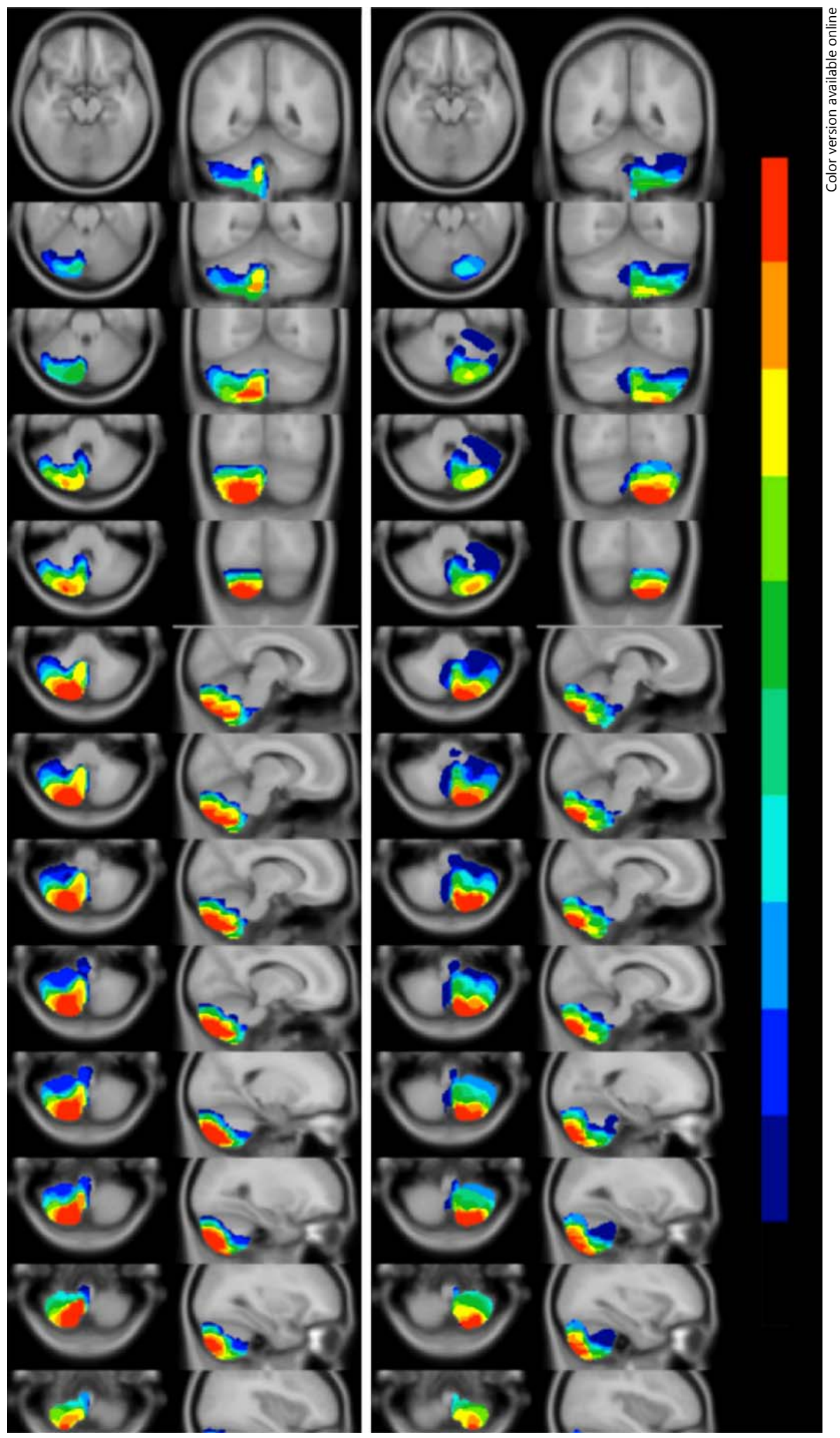

Eur Neurol 2017;77:137-146 

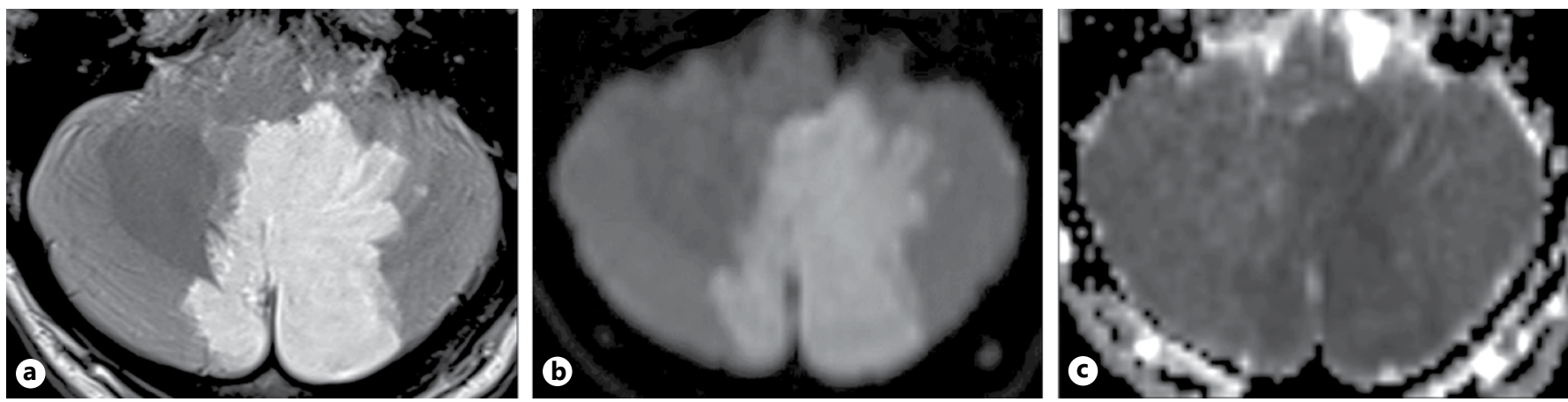

Fig. 3. Acute PICA infarct involving both hemispheres seen on T2WI (a), DWI (b), and ADC-map (c). Not rarely, PICA territories may cross the midline. As its name implies, the PICA supplies the posterior inferior surface of the cerebellum. Notice, however, that the lateral portions of the posterior inferior surfaces are not involved.
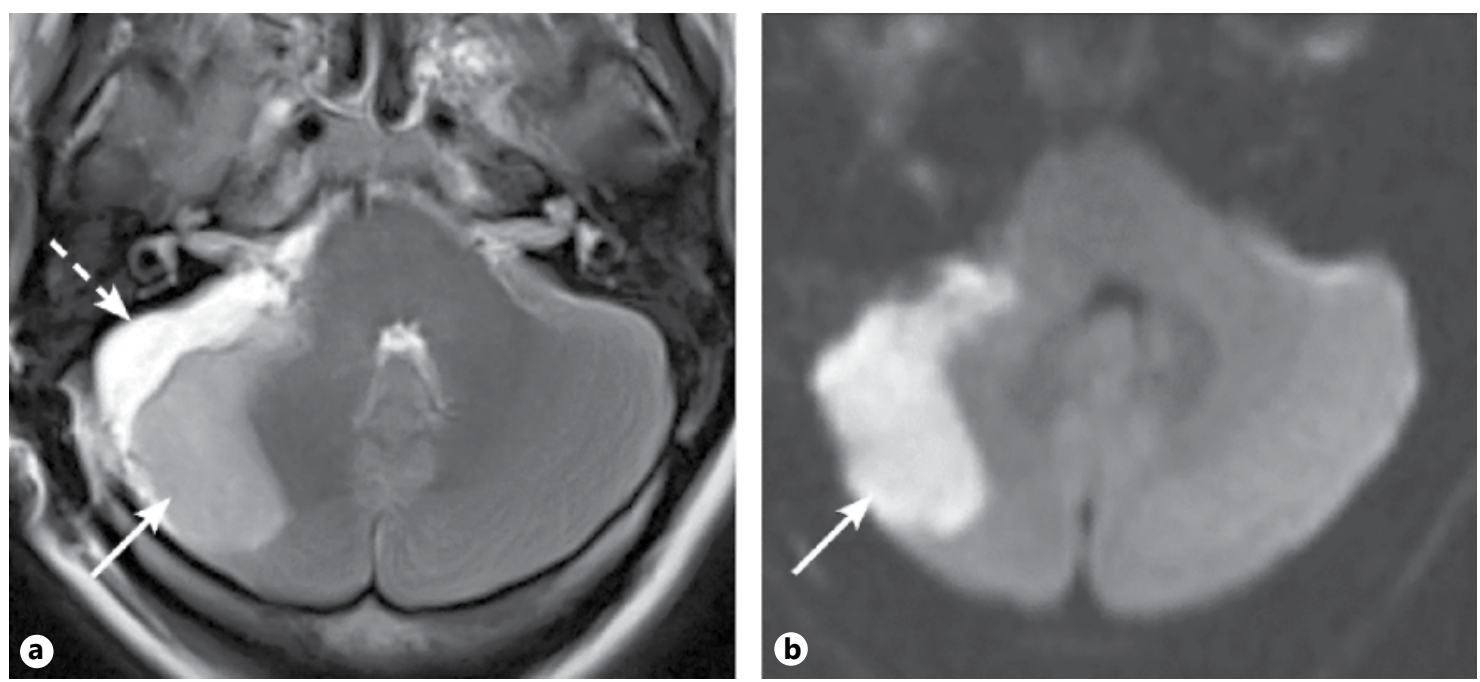

Fig. 4. Acute AICA infarction (arrows) immediately after surgery for acoustic schwannoma on T2WI (a) and DWI (b). The AICA often shows a loop in the inner auditory canal, and is therefore at risk during surgery. This case highlights how AICA territories may extend beyond the lateral angle to supply the lateral portion of the posterior cerebellar surface, not to be confused with PICA infarction. Also visible is a post-operative pseudomeningocele in between the petrous bone and the right cerebellar hemisphere (dashed arrow). the territory of the superior cerebellar artery often includes the upper part of the pontine tegmentum [14].

Branches of the PICA, AICA and superior cerebellar artery (SCA) run on the surface of the cerebellum, while more peripheral branches run in the cerebellar fissures and give rise to cortical arteries. Anastomoses between peripheral branches of the PICA, AICA, and SCA are consistently present among individuals $[13,15,16]$. The territory perfused by the AICA varies in reciprocity with the PICA and SCA $[13,16]$. It may be confined to the anterior surface, but the AICA may also supply lateral portions of the posterior and/or superior surface of the cerebellum (Fig. 4). Nevertheless, even in extreme domi- nance of the AICA over the PICA (AICA-PICA variant), a small and possibly unrecognisable PICA limited to its medullary territory exists. As a result, even dominant AICA strokes do not produce lateral medullary infarcts (or Wallenberg syndromes). A PICA-AICA is characterised by a small AICA and large PICA cortical supply. SCA can dominate as well, and for instance, supply the inferior vermian territory in addition to the superior vermis.

Recently, the cerebellar PICA-perfusion territories have been made visible in vivo in individual subjects by the use of super-selective (territorial) arterial spin labeling (ASL) MRI, which is a non-invasive technique to measure brain perfusion without the need to administer 

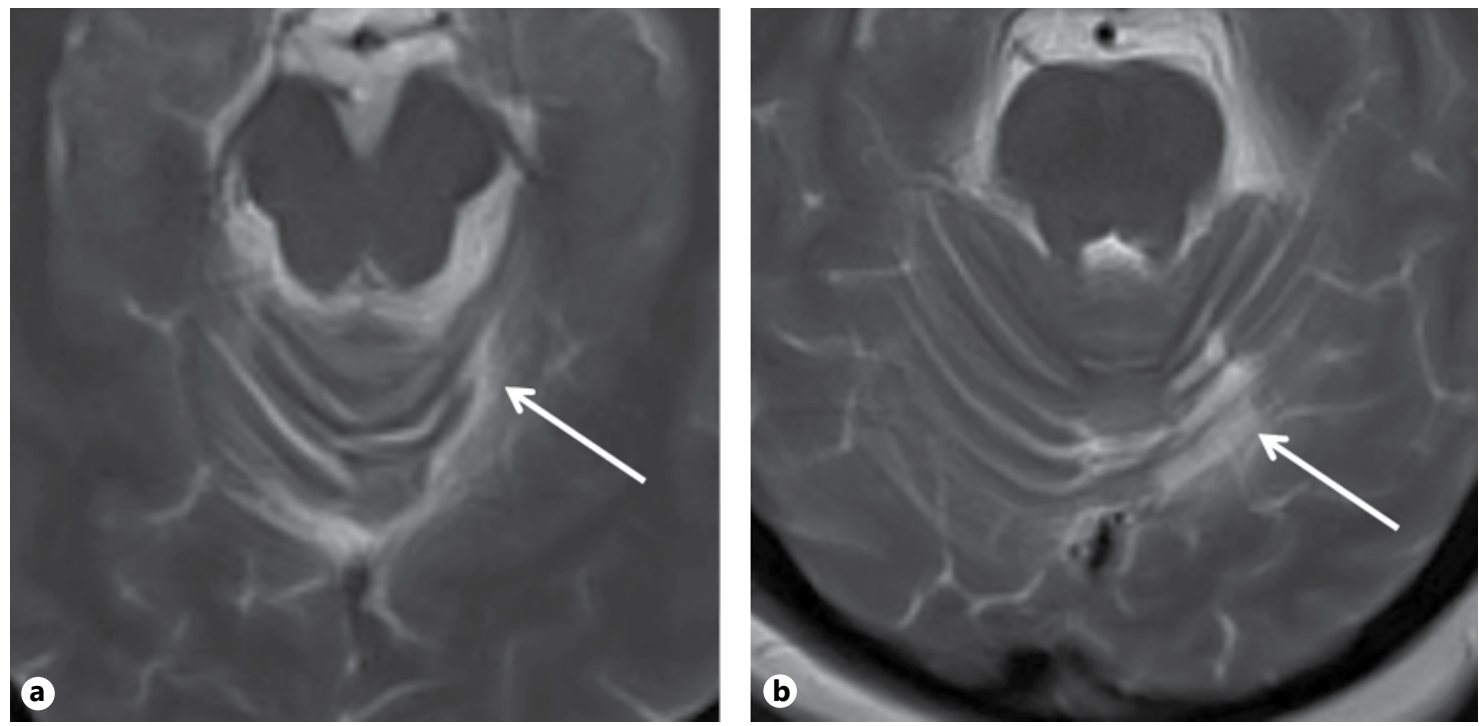

Fig. 5. Old SCA infarction involving the anterior lobe of the left cerebellar hemisphere on T2WI at the level of the midbrain (a) and superior cerebellar peduncles (b).

an exogenous contrast agent [17]. In ASL, arterial blood water is magnetically labelled and then imaged, and with selective labeling, the arterial perfusion territories can be visualised [17-19]. By labelling both vertebral arteries, the territories supplied by one vertebral artery (PICA) can be distinguished from those supplied by both vertebral arteries (AICA and SCA) and from the contralateral vertebral artery (contralateral PICA) (Fig. 2) [13]. Since the PICA is the only artery originating from the vertebral artery, ASL signal within the PICA-territory is only observed with labelling of the ipsilateral vertebral artery, while labelling of the contralateral vertebral artery results in a signal void. Although the technique has so far only been studied in a research setting, the application of the described technique already allowed to create a probabilistic flow territory map of the PICA-perfusion territory based on 10 healthy subjects, as illustrated in Fig. 1 [13]. We foresee that in the future, this technique will allow to correlate small cerebellar infarcts with cerebellar arterial perfusion territories, as has recently also been shown for infarcts and perfusion territories in the cerebrum [19].

\section{Pathophysiological Mechanisms}

\section{Larger Infarcts}

Larger cerebellar infarcts (diameter $>1.5 \mathrm{~cm}$ ) typically affect one or more of the 3 major cerebellar perfusion territories (PICA, AICA, and SCA), either in whole or in part. Therefore, these infarcts have been referred to as territorial infarcts (Fig. 1, 3-5) [11]. They are caused by the occlusion of a major cerebellar artery (PICA, AICA, and/or SCA), or from a proximal branch stemming from it. Arterial occlusion usually results from thromboembolism from the heart or an arterial source (mainly atherosclerosis and less frequently dissection) [20].

\section{Small Infarcts and Microinfarcts}

Small cerebellar infarcts (diameter $\leq 1.5 \mathrm{~cm}$ ) have been recently found to be far more common than larger infarcts, and they tend to present on imaging studies as an incidental finding rather than being detected in the acute or symptomatic stage of infarction [5], either because of mild clinical symptoms $[21,22]$ or because of the fact that MRI is not timely performed (beyond the stage of diffusion restriction). A vast majority of small cerebellar infarcts are located in the cortex and are of atherosclerotic and/or thromboembolic origin, with or without associated low blood flow [11, 23]. Since these small infarcts typically occur in a distal (cortical) arterial territory, they are sometimes referred to as end-territorial infarcts $[11,12]$, while the smallest of them have become recently known as cortical cerebellar microinfarcts (CCeMIs) [24]. Although CCeMIs have so far only been studied on postmortem 7T MRI studies, they are also visible in the DWI positive acute stage on routine MRI studies in vivo $[25,26]$. More rarely, small infarcts may occur in the deep subcortical regions of the cerebellum to affect the deep 
white matter and/or deep nuclei $[5,23,27]$. Such small deep infarcts may be related to small vessel disease in rare cases $[12,23]$. Lesions restricted to the juxtacortical white matter are usually not of ischemic origin [5].

\section{Venous Ischaemia}

Cerebellar venous infarction or haemorrhage due to isolated venous thrombosis of the posterior fossa is a rare form of intracranial vein thrombosis that can be unsuspected in clinical practice. Common risk factors for venous sinus thrombosis include puerperium, oral contraceptives, protein $\mathrm{C}$ of factor $\mathrm{V}$ Leiden deficiency and dehydration [28-30].

\section{MRI}

\section{Pattern Recognition}

In general, diagnosis of large cerebellar infarcts is more straightforward than diagnosis of small infarcts. Large cerebellar infarcts usually do not cause diagnostic difficulties because of their vascular distribution in a typical perfusion territory, which is easily recognised and reflects the name of the cerebellar artery by which it is supplied. The inferior part of the posterior cerebellar surface is supplied by the PICA, the inferior part of the anterior surface by the AICA, and the superior surface by the SCA. Therefore, for large cerebellar infarcts, the involved perfusion territories are usually easily deduced from the cerebellar surface (anterior, posterior, or superior) bordering the infarct. Often, however, the AICA supplies a considerable part of the posterior surface (Fig. 4). Nevertheless, the medial part of the posterior surface is (almost) invariably supplied by the PICA (Fig. 1-3), while its lateral extension is far more variable (Fig. 1) [13]. Thus, so-called medial (branch) PICA infarcts may result from infarction of an entire cerebellar PICA territory restricted to the medial part of the cerebellum (Fig. 1-3) [13, 31]. Likewise, AICA infarcts extending into the lateral portions of the posterior surface (Fig. 4) should not be mistaken for lateral PICA infarcts. Other variants to consider include a combined PICA-AICA territory (with a common trunk seen on angiographic sequences) or a PICA supplying both cerebellar hemispheres (Fig. 3).

Importantly, brainstem infarcts associated with cerebellar infarcts are helpful to define which cerebellar artery is involved, and may confirm that the medial branch of this artery participates in the infarction (Fig. 1) [32,33].

Of note also is that multiple acute infarcts in the posterior circulation usually involve the cerebellum, and that simultaneous brainstem and posterior cerebral artery territory infarcts sparing the cerebellum are uncommon [34].

Small cerebellar infarcts are only rarely detected in the acute stage, unless in patients with multiple cerebellar infarctions (Fig. 6) or in patients with simultaneous infarcts in the brainstem or cerebrum $[12,35]$. As a result, small cerebellar infarcts often present later in life as an incidental cavity on imaging studies (Fig. 7) [7, 8, 27]. Most of these are cerebellar cortical infarct cavities, which spare the subjacent branches of subcortical white matter (Fig. 7) [5, 7, 27]. In the axial plane, small cortical infarcts are usually oriented/radiated towards the brain stem (Fig. 7) [5]. In the axial and sagittal plane, small cortical infarcts are located along cerebellar fissures (Fig. 7c) [5].

\section{MRI Sequences and Infarct Evolution}

Acute cerebellar infarcts (Fig. 1, 3, 4, and 6) are usually readily recognised as bright areas on DWI with low signal on apparent diffusion coefficient (ADC) maps due to restricted diffusion, although it should be noted that infarcts in the posterior circulation may be more often DWI negative than those in the anterior circulation [3638]. DWI becomes positive within minutes after the onset of infarction because of cytotoxic oedema $[1,30]$. In the following hours, infarcts become hyperintense on FLAIR and T2-weighted images (T2WI). Nevertheless, T2WI are preferred as FLAIR images are often false negative in the posterior fossa due to local field heterogeneities [4]. Adding time-of-flight (TOF) MR angiography (MRA) or contrast-enhanced dynamic MRA of the head and neck may help to visualise arterial variations and any underlying arterial lesion that may be the cause of the infarction (Fig. 1) [20, 34, 39].

Brain swelling peaks at about 3 days, and infarct volume in the acute stage on DWI is therefore not a reliable indicator of the final infarct size [30]. In the second half of the first week, infarcts considerably diminish in size and diffusion (pseudo-)normalises after around 10 days (1-4 weeks) [30]. Thus, MRI provides the greatest diagnostic yield when performed as soon as possible (certainly within a few days) of symptom onset, especially in minor stroke. Also around 10 days, (subacute) infarcts may become isointense and therefore (nearly) invisible on different MRI sequences, a phenomenon known as "fogging," which corresponds to the occurrence of necrosis, angiogenesis and microglia/macrophage infiltration [40-42]. In this stage, infarcts may be best seen as areas of parenchymal contrast enhance- 

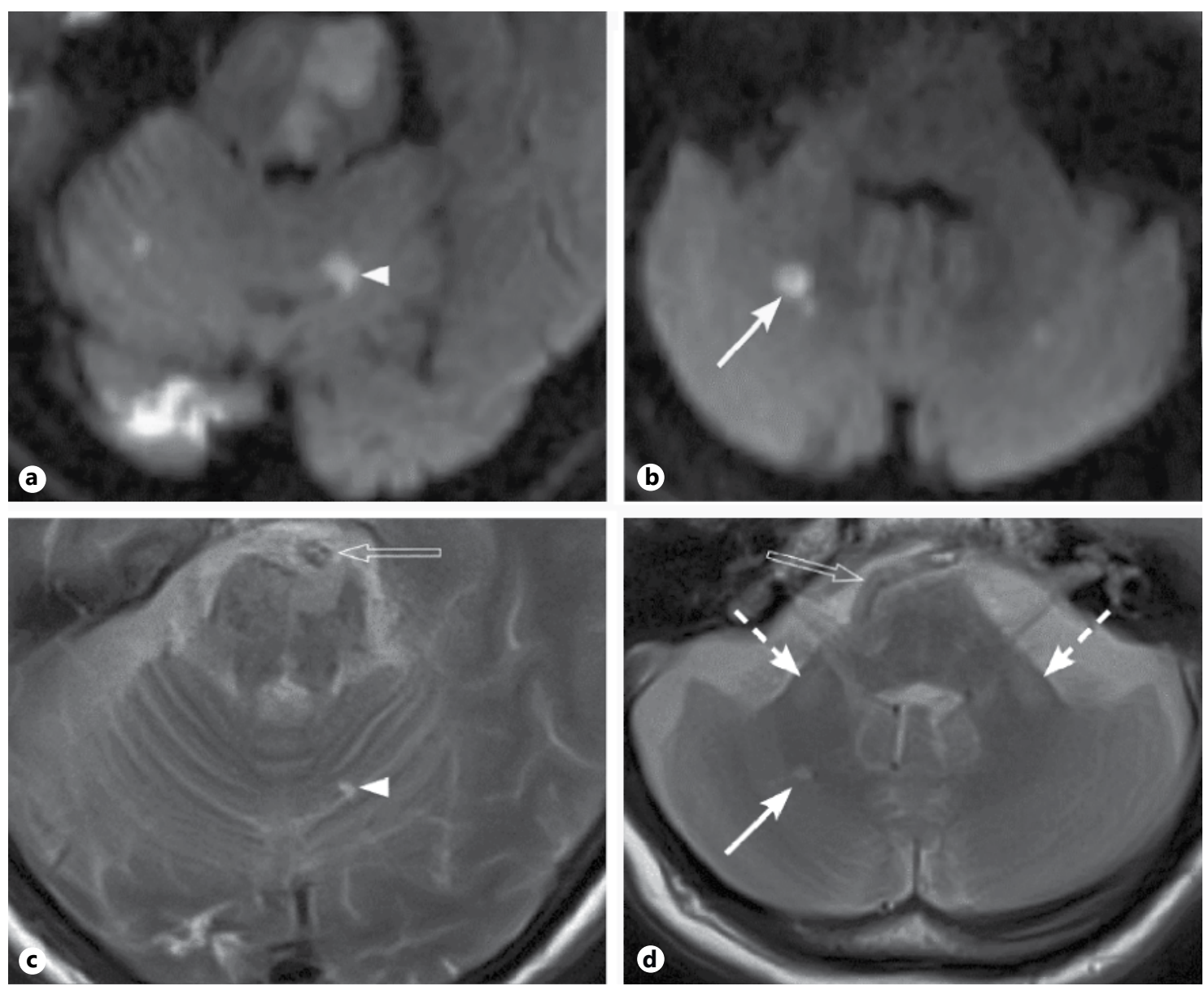

Fig. 6. Initial DWI (a, b) show acute left pontine and right occipital lobe infarction as well as 3 small acute infarcts in the right and 2 in the left cerebellar hemispheres. The largest cerebellar infarct on the right (arrow) measures $0.7 \mathrm{~cm}$ and on the left (arrowhead) $0.8 \mathrm{~cm}$, while the others are smaller than $0.5 \mathrm{~cm}$. Follow-up T2WI (c, d) more than half a year later again show the pons infarct as well as an occluded basilar artery (open arrow). Due to infarct retraction, only the 2 largest cerebellar infarcts are still visible. T2-hyperintense changes appear in both middle cerebellar peduncles due to Wallerian degeneration of white matter tracts (dashed arrows).
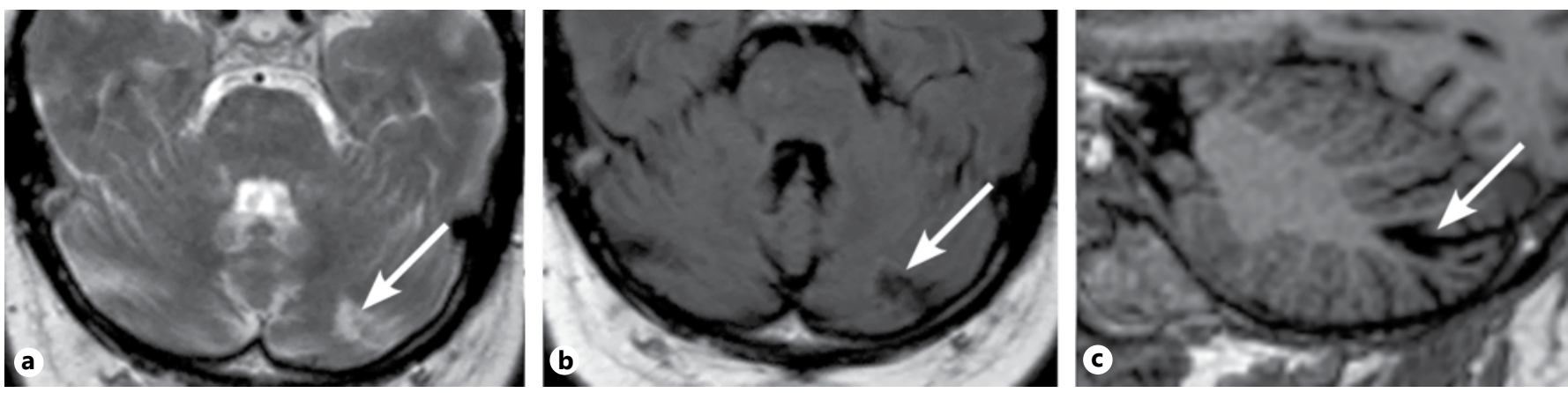

Fig. 7. Cerebellar cortical infarct cavity (arrows) in the left posterior lobe along the great horizontal fissure. Notice the radial orientation towards the brain stem on axial T2 (a) and FLAIR images (b). Some hyperintense signal (gliosis) surrounding the cavity is seen (b). Notice the cortical location in the apex of the fissure on sagittal reconstruction of 3D T1-weighted dataset (c), with strict sparing of subjacent white matter. 

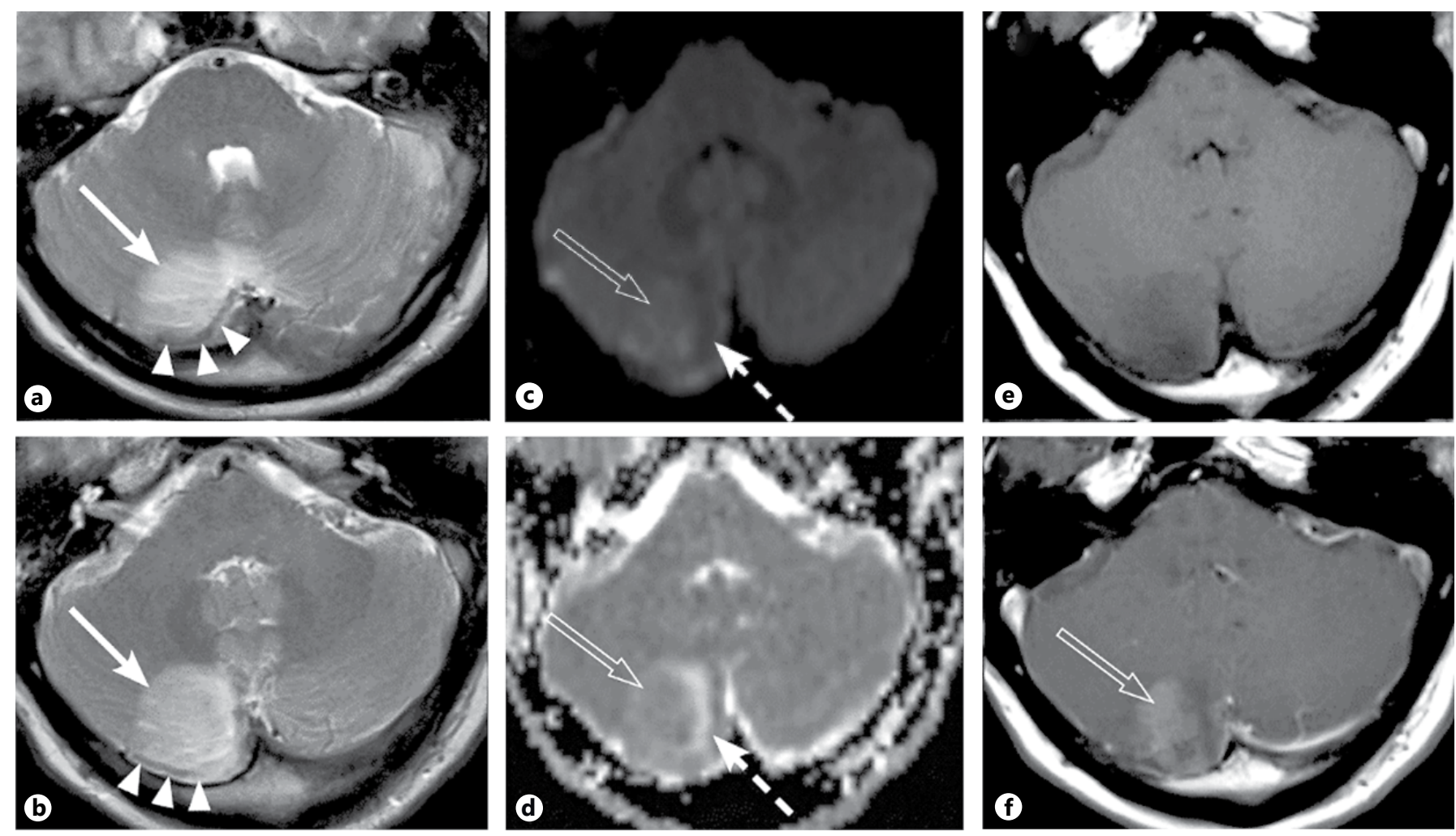

Fig. 8. Axial T2WI (a, b) show thrombosis of the right lateral sinus (arrowheads) complicated by venous infarction (arrows) in the right cerebellar hemisphere. The infarct core (open arrows) shows discretely restricted diffusion on DWI (c) and ADC map (d), and is surrounded by a rim of vasogenic oedema with facilitated diffusion (dashed arrows). Axial T1WI before (e) and after (f) the administration of gadolinium show faint enhancement of the infarct core (open arrow). ment, which appears around 1 week and may be visible up to 8 weeks to 4 months after infarction. Afterwards, both small and large cerebellar infarcts tend to heal with cavitation, leaving a cerebrospinal fluid-filled cavity (Fig. 5, 6c, d, 7) surrounded by a rim of gliosis [27]. It has been shown that high-resolution T1-weighted images (T1WI) are superior to FLAIR images for confirming infarct cavitation suspected on T2WI; low intensities equal to CSF reflecting cavitation and less pronounced hypo-intensities reflecting gliosis [4]. Nevertheless, if present, a hypo-intense (fluid-filled) cavity with a hyperintense rim on FLAIR is diagnostic of cavitation with surrounding gliosis (Fig. 7b) $[4,5]$. As described above, small cerebellar infarcts are often only detected as an incidental infarct cavity, which are most often smaller than $1 \mathrm{~cm}$ [5]. Cortical cerebellar infarcts smaller than $5 \mathrm{~mm}$ in the DWI positive stage tend to become imperceptible on successive clinical MRIs (Fig. 6). These infarcts also include CCeMIs, which are visible on clinical MRIs only in the DWI positive stage and later on exclusively on (ultra-)high-field MRI [7, 24, $27,26]$. Nevertheless, CCeMIs have so far almost exclusively been investigated on postmortem studies $[24,26$, 43].

\section{Venous Ischaemia}

Cerebellar venous infarction usually results from thrombosis of the straight sinus, lateral sinuses or superior petrosal vein $[28,29]$. MRI can show the causal thrombosis as well as the parenchymal abnormalities. The parenchymal abnormalities consist of ischaemic/oedematous lesions in a non-arterial distribution. DWI may be positive or negative, and contrast enhancement may be present (Fig. 8). Also there is a tendency for haemorrhagic alterations that may be visible on $\mathrm{T} 2 *$ or susceptibility-weighted imaging (SWI) images [44]. Although venous clot may be visible directly on T1WI or T2WI as absent flow voids or on DWI as a zone of restricted diffusion in the vessel lumen, contrast-enhanced MRI with the inclusion of MR venography is the imaging study of 
choice for diagnosing sinus thrombosis $[28,30]$. If unenhanced MRI alone is used, thrombus in the early stage of evolution may mimic normal flow [29]. SWI is also a good technique to demonstrate venous sinus thrombosis, since deoxyhemoglobin within thrombus can be detected on T2* and especially SWI by the presence of hypointensity and blooming [44].

\section{Conclusion}

For optimal patient care, clinicians should be familiar with the presentation of cerebellar infarctions on MRI, which should be performed within the slightest delay as possible. In the acute stage, DWI allows for excellent visualisation of acute cerebellar ischaemia. Nevertheless, be- cause of non-specific clinical findings and/or delays in MRI, many cerebellar infarcts are still only detected after healing as incidental cerebellar infarct cavities. These show typical imaging characteristics and are often the only way to identify foregoing cerebellar infarction in the individual patient.

\section{Acknowledgements}

This work was supported by the European Research Council, ERC grant agreement No. 637024 (J.H.). This work was supported by The Netherlands Organization for Scientific Research (NWO) under grant No. 91712322 (J.H.).

\section{Disclosure Statement}

The authors report no conflict of interest.

\section{References}

1 van Everdingen KJ, van der Grond J, Kappelle LJ, et al: Diffusion-weighted magnetic resonance imaging in acute stroke. Stroke 1998; 29:1783-1790.

2 Read SJ, Jackson GD, Abbott DF, et al: Experience with diffusion-weighted imaging in an acute stroke unit. Cerebrovasc Dis 1998;8: 135-143.

3 Redgrave JN, Schulz UG, Briley D, et al: Presence of acute ischaemic lesions on diffusionweighted imaging is associated with clinical predictors of early risk of stroke after transient ischaemic attack. Cerebrovasc Dis 2007; 24:86-90.

4 Moreau F, Patel S, Lauzon ML, et al: Cavitation after acute symptomatic lacunar stroke depends on time, location, and MRI sequence. Stroke 2012;43:1837-1842.

5 De Cocker LJ, Geerlings MI, Hartkamp NS, et al: Cerebellar infarct patterns: the SMART-Medea study. NeuroImage Clin 2015;8:314-321.

6 Compter A, Kappelle LJ, Algra A, van der Worp HB: Nonfocal symptoms are more frequent in patients with vertebral artery than carotid artery stenosis. Cerebrovasc Dis 2013; 35:378-384.

7 De Cocker LJ, Kloppenborg RP, van der Graaf $Y$, et al: Cerebellar cortical infarct cavities: correlation with risk factors and MRI markers of cerebrovascular disease. Stroke 2015;46: 3154-3160.

8 De Cocker LJ, Compter A, Kappelle LJ, et al: Cerebellar cortical infarct cavities and vertebral artery disease. Neuroradiology 2016;58: 853-857.

9 Datar S, Rabinstein AA: Cerebellar infarction. Neurol Clin 2014;32:979-991.

10 Edlow JA, Newman-Toker DE, Savitz SI: Diagnosis and initial management of cerebellar infarction. Lancet Neurol 2008;7:951-964.
11 Amarenco P, Lévy C, Cohen A, et al: Causes and mechanisms of territorial and nonterritorial cerebellar infarcts in 115 consecutive patients. Stroke 1994;25:105-112.

12 De Cocker LJ, van Veluw SJ, Fowkes M, Luijten PR, Mali WP, Hendrikse J: Very small cerebellar infarcts: integration of recent insights into a functional topographic classification. Cerebrovasc Dis 2013;36:8187.

13 Hartkamp NS, De Cocker LJ, Helle M, et al: In vivo visualization of the PICA perfusion territory with super-selective pseudo-continuous arterial spin labeling MRI. Neuroimage 2013;83:58-65.

14 Tatu L, Moulin T, Bogousslavsky J, Duvernoy $\mathrm{H}$ : Arterial territories of human brain: brainstem and cerebellum. Neurology 1996;47: 1125-1135.

15 Marinković S, Kovacević M, Gibo H, et al: The anatomical basis for the cerebellar infarcts. Surg Neurol 1995;44:450-460; discussion 460-461.

16 Icardo JM, Ojeda JL, Garcia-Porrero JA, Hurle JM: The cerebellar arteries: cortical patterns and vascularization of the cerebellar nuclei. Acta Anat 1982;113:108-116.

17 Bokkers RP, De Cocker LJ, van Osch MJ, et al: Selective arterial spin labeling: techniques and neurovascular applications. Top Magn Reson Imaging 2016;25:73-80.

18 Kansagra AP, Wong EC: Mapping of vertebral artery perfusion territories using arterial spin labeling MRI. J Magn Reson Imaging 2008;28:762-766.

19 Hartkamp NS, Hendrikse J, De Cocker LJL, et al: Misinterpretation of ischaemic infarct location in relationship to the cerebrovascular territories. J Neurol Neurosurg Psychiatry 2016;87:1084-1090.
20 Bogousslavsky J, Regli F, Maeder P, et al: The etiology of posterior circulation infarcts: a prospective study using magnetic resonance imaging and magnetic resonance angiography. Neurology 1993;43:1528-1533.

21 Saini M, Ikram K, Hilal S, et al: Silent stroke: not listened to rather than silent. Stroke 2012; 43:3102-3104.

22 Fanning JP, Wesley AJ, Wong AA, Fraser JF: Emerging spectra of silent brain infarction. Stroke 2014;45:3461-3471.

23 Park KY, Chung PW, Kim YB, et al: Association between small deep cerebellar ischemic lesion and small-vessel disease. Cerebrovasc Dis 2009;28:314-320.

24 De Reuck JL, Deramecourt V, Auger F, et al: The significance of cortical cerebellar microbleeds and microinfarcts in neurodegenerative and cerebrovascular diseases. A postmortem 7.0-tesla magnetic resonance study with neuropathological correlates. Cerebrovasc Dis 2015;39:138-143.

25 Gass A, Ay H, Szabo K, Koroshetz WJ: Diffusion-weighted MRI for the "small stuff": the details of acute cerebral ischaemia. Lancet Neurol 2004;3:39-45.

26 De Cocker LJ, Lindenholz A, Zwanenburg JJ, et al: Clinical vascular imaging in the brain at $7 \mathrm{~T}$. Neuroimage 2016; pii:S1053-8119(16)30661-9.

27 De Cocker LJ, van Veluw SJ, Biessels GJ, Spliet WGM, Thunnissen IE, Luijten PR, Hendrikse J, Zwanenburg JJ: Ischaemic cavities in the cerebellum: an ex vivo 7-tesla MRI study with pathological correlation. Cerebrovasc Dis 2014;38:17-23.

28 Ruiz-Sandoval JL, Chiquete E, Navarro-Bonnet $\mathrm{J}$, et al: Isolated vein thrombosis of the posterior fossa presenting as localized cerebellar venous infarctions or hemorrhages. Stroke 2010;41:2358-2361. 
29 Hacein-Bey L, Varelas PN, Ulmer JL, et al: Imaging of cerebrovascular disease in pregnancy and the puerperium. AJR Am J Roentgenol 2016;206:26-38.

30 Kanekar SG, Zacharia T, Roller R: Imaging of stroke: part 2, pathophysiology at the molecular and cellular levels and corresponding imaging changes. AJR Am J Roentgenol 2012; 198:63-74.

31 Amarenco P, Roullet E, Hommel M, et al: Infarction in the territory of the medial branch of the posterior inferior cerebellar artery. J Neurol Neurosurg Psychiatry 1990;53:731735.

32 Barth A, Bogousslavsky J, Regli F: Infarcts in the territory of the lateral branch of the posterior inferior cerebellar artery. J Neurol Neurosurg Psychiatry 1994;57:1073-1076.

33 Vuilleumier P, Bogousslavsky J, Regli F: Infarction of the lower brainstem. Clinical, aetiological and MRI-topographical correlations. Brain 1995; 118(pt 4):10131025.
34 Bernasconi A, Bogousslavsky J, Bassetti C, Regli F: Multiple acute infarcts in the posterior circulation. J Neurol Neurosurg Psychiatry 1996;60:289-296.

35 Canaple S, Bogousslavsky J: Multiple large and small cerebellar infarcts. J Neurol Neurosurg Psychiatry 1999;66:739-745.

36 Oppenheim C, Stanescu R, Dormont D, et al: False-negative diffusion-weighted MR findings in acute ischemic stroke. AJNR Am J Neuroradiol 2000;21:1434-1440.

37 Nouh A, Remke J, Ruland S: Ischemic posterior circulation stroke: a review of anatomy, clinical presentations, diagnosis, and current management. Front Neurol 2014;5:30.

38 Makin SD, Doubal FN, Dennis MS, Wardlaw JM: Clinically confirmed stroke with negative diffusion-weighted imaging magnetic resonance imaging: longitudinal study of clinical outcomes, stroke recurrence, and systematic review. Stroke 2015;46:3142-3148.
39 Akgun V, Battal B, Bozkurt Y, et al: Normal anatomical features and variations of the vertebrobasilar circulation and its branches: an analysis with 64-detector row CT and 3T MR angiographies. ScientificWorldJournal 2013; 2013:620162.

40 Scuotto A, Cappabianca S, Melone MB, Puoti G: MRI "fogging" in cerebellar ischaemia: case report. Neuroradiology 1997;39:785-787.

41 O'Brien P, Sellar RJ, Wardlaw JM: Fogging on T2-weighted MR after acute ischaemic stroke: how often might this occur and what are the implications? Neuroradiology 2004;46:635-641.

42 Wagner DC, Deten A, Härtig W, et al: Changes in $\mathrm{T} 2$ relaxation time after stroke reflect clearing processes. Neuroimage 2012;61:780-785.

43 Arvanitakis Z, Leurgans SE, Barnes LL, et al: Microinfarct pathology, dementia, and cognitive systems. Stroke 2011;42:722-727.

44 Heyn C, Alcaide-Leon P, Bharatha A, et al: Susceptibility-weighted imaging in neurovascular disease. Top Magn Reson Imaging 2016; 25:63-71 